\title{
Experimental study of guided waves propagation characteristics under the changing temperatures
}

\author{
Xixi Yan', Lei Qiu², Shenfang Yuan ${ }^{3}$ \\ Nanjing University of Aeronautics and Astronautics, Nanjing, China \\ ${ }^{2}$ Corresponding author

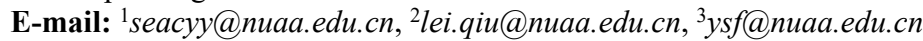 \\ Received 26 September 2018; accepted 1 October 2018 \\ DOI https://doi.org/10.21595/vp.2018.20262
}

Check for updates

Copyright (C) 2018 Xixi Yan, et al. This is an open access article distributed under the Creative Commons Attribution License, which permits unrestricted use, distribution, and reproduction in any medium, provided the original work is properly cited.

\begin{abstract}
Structural Health Monitoring (SHM) plays an important role in ensuring the safe use of aerospace, architecture and other fields. At present, SHM based on piezoelectric and guided waves is a hot spot for real-time monitoring and nondestructive testing. However, the effect of environmental temperatures may mask the signal changes caused by damage and lead to failure of guided wave SHM methods. In this paper, the influence mechanism of the changing temperatures on the propagation characteristics of the guided wave is analyzed. Piezoelectric constants changed with the changing temperatures due to the amplitude variation of the guided wave with the changing temperatures. The effect of temperature on material properties of the plate structures will result in the influence of the guided wave propagation speed. An experiment is performed to study the propagation characteristics of the guided wave on an aluminium plate under changing temperatures. The experimental results indicate that the temperature can change the guide wave amplitude and velocity. At the same time, the temperature effect has the same variation trend at different frequencies, and the slopes of the linear fitting of the propagation characteristics of guided waves with the changing temperatures are also almost consistent.
\end{abstract}

Keywords: structural health monitoring, guided wave, changing temperatures, piezoelectric constants, the material properties of the plate structures.

\section{Introduction}

In recent years, a growing number of theoretical research work and practical application in engineering of SHM based on piezoelectric and guided waves, are the results of the advantages about guided waves in non-destructive real-time monitoring gradually understood and used. In a SHM method based on piezoelectric guided waves, the piezoelectric sensor is used to actuate and sense the guided wave signals, which is often pasted on the surface of the structures or embedded into the internal [1], and some guided wave integrated monitoring systems in signal acquisition $[2,3]$ gradually developed that will be conducive to the application in engineering practice. The piezoelectric wafers used in the damage detection method has the advantages of fast response speed, low power consumption, low cost and small size, resulting in especially suitable for integrated into structures. Guided waves are sensitive to small damage and can achieve regional monitoring [4, 5]. Since the method is based on the interaction between guided waves and damage, the propagation of guided waves will also be affected by environmental factors when the engineering is monitored in the actual working environment.

It is well known that aerospace structures conducted real-time on-line SHM are usually subjected to changing temperatures and damage at the same time. The environmental changing temperatures will directly affect the sensor signal features, resulting in the damage diagnosis is not carried out reliably. Therefore, how to guarantee the reliability of damage monitoring under time-varying environment is a key problem in the practical application of SHM technology. From the present research reports, based on the piezoelectric and guided waves SHM affected by the temperatures in the wave amplitude is mainly reflected on the piezoelectric sensor-electric transformation characteristics under changing temperatures [6-9]. According to Raghavan and 
Cesnik's research results, by fitting the piezoelectric constant $d_{31}$ curve under changing temperatures, bring the obtained $d_{31}$ into the piezoelectric equation to carry out the whole process simulation of guided wave excitation - propagation - response. The propagation velocity will vary with temperatures due to the change of the guide wave velocity is mainly determined by the influence of the material properties of the structures under changing temperatures $[9,10]$. However, there are few researches on the effect of changing temperatures on the piezoelectric parameters and the combined influence of piezoelectric and guided waves in the real monitoring system. Therefore, this paper discusses the influence mechanism of piezoelectric and guided wave by temperatures, designs and implements the experiment to find out the effect of the changing temperatures on the propagation of guided wave based on piezoelectric wafers in the thin plate.

Based on the above discussion, we arrange this paper content is as follows: in the second part, discuss the influence mechanism of temperatures on piezoelectric and guided waves, and introduce the experiment and the implementation process according to the mechanism. In the third part, get the experimental results from the experimental data processing, and analyze the experimental results; In the fourth part, summarize the conclusions and make a certain prospect.

\section{Experiment principles, settings and process under the changing temperatures}

\subsection{Experiment principles under the changing temperatures}

Taking the guided wave propagation of the simplest flat sheet structure under the changing temperatures as an example to illustrate the experimental principles of temperature effect, the schematic diagram of the temperature and guided wave acting on the structure is shown in Fig. 1. In the process of excitation-propagation-response, the influence mechanism of the temperatures is summarized as following four aspects [9, 11-13]:

(1) The changing temperatures effect on excitation and response performance of piezoelectric wafers: This effect is mainly reflected in the change of electro-mechanical transform characteristics of piezoelectric ceramics under the changing temperatures, and accompanied with thermal expansion, which leads to the changes of the excitation and response characteristics of guided wave, causing the change of the guided waves in the amplitude.

(2) The changing temperatures effect on the coupling properties of the bond: The adhesive only acts as a shear force, and very thin, so the impact of the temperature on the bond can temporarily not be considered.

(3) The changing temperatures effect on the propagation of the guided waves: This effect is mainly reflected in the changes of material properties when the structure is subjected to the changing temperature and accompanied by thermal expansion, leading to changes in the velocity of guided waves, causing the change of the guided waves in the time of flight (TOF).

(4) As the thermal expansion coefficient of the structure, bond and piezoelectric ceramics is inconsistent, the stress concentration of the piezoelectric ceramics will be caused when the thermal expansion is generated, thus changing the machine-to-electrical conversion characteristics of piezoelectric ceramics. This is equivalent to the effect of external loads on piezoelectric ceramics.

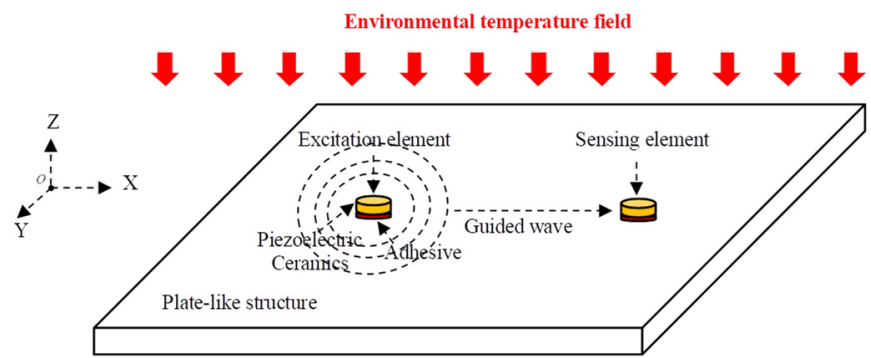

Fig. 1. Transmission schematic diagram of guided wave in the structure under changing temperatures 
In order to get more thorough study about temperature effect and according to the above mechanism of the changing temperatures, we can set the influence of temperatures on the propagation characteristics of guided wave to study the influence law about environmental factors on the amplitude and propagation velocity of Lamb wave signal in aluminum plate.

\subsection{Experiment settings and process}

In this experiment, the Lamb wave signals in the aluminum plates subjected to changing temperatures are collected. The PXI scanning system is used to collect the Lamb wave signal; a 2024 aluminum plate with piezoelectric wafers is mounted. The specimen size is $510 \mathrm{~mm} \times 510 \mathrm{~mm} \times 3 \mathrm{~mm}$, and the material properties are: Young's modulus $70 \mathrm{GPa}$, density $2700 \mathrm{~kg} / \mathrm{m}^{3}$, Poisson's ratio 0.33 . Fig. 2 shows the overall the test device and the specimen setting in an environmental chamber. The specific size of the specimen and the placement of the piezoelectric wafers are shown in Fig. 3.

In this experiment, the temperature rose every $5^{\circ} \mathrm{C}$ from $-20^{\circ} \mathrm{C}$ to $40^{\circ} \mathrm{C}$, and every $2{ }^{\circ} \mathrm{C}$ from $40^{\circ} \mathrm{C}$ to $60^{\circ} \mathrm{C}$, a total number of 23 temperatures, according to the temperature range commonly used in aircraft. The lamb wave signals by frequency scanning are obtained at the different but stable temperatures.

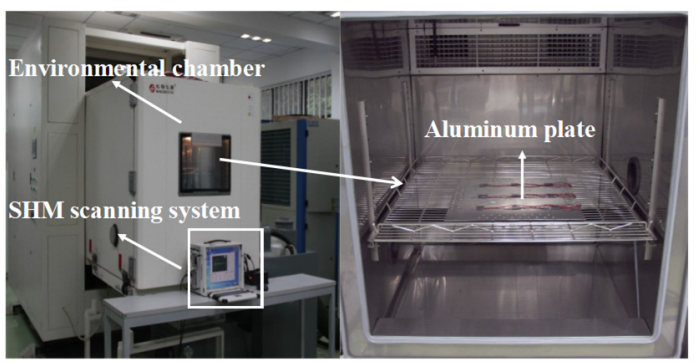

Fig. 2. A schematic diagram of a vibration separator

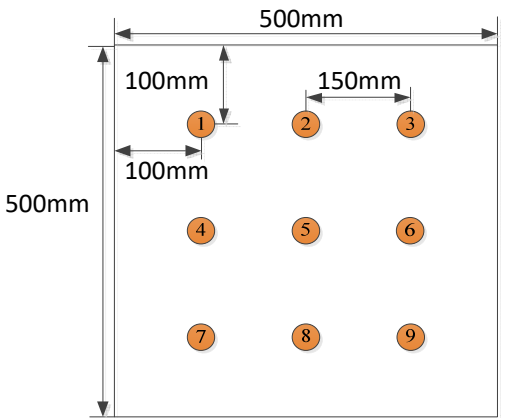

Fig. 3. Specimen size and piezoelectric wafers layout drawing

\section{Experimental results and discussion of temperature effect}

Due to the symmetry of specimen size, According to the experimental purpose, the signal of typical channel 2-5 (piezoelectric wafer number) is selected.

Firstly, Figs. 4-7 shows the center frequencies from 50 to $250 \mathrm{kHz}$ how $S_{0}$ mode and $A_{0}$ mode change of amplitude and TOF under changing temperatures. Measurements of TOF changes are used to indirectly measure the group velocity changes.

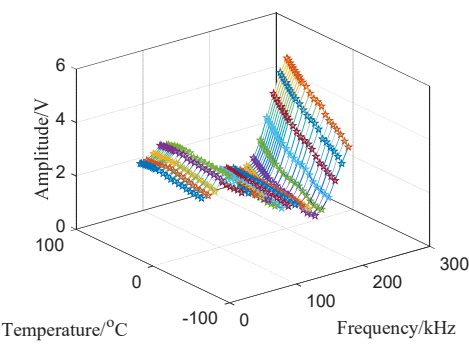

a) Scatter plot-50 to $250 \mathrm{kHz}$

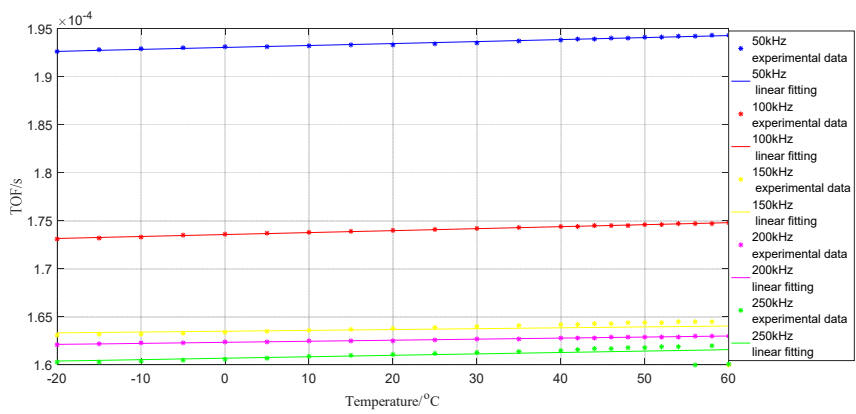

b) Plot-5 typical frequencies

Fig. 4. Influence of changing temperatures on $S_{0}$ amplitude at actuator 2 to sensor 5 


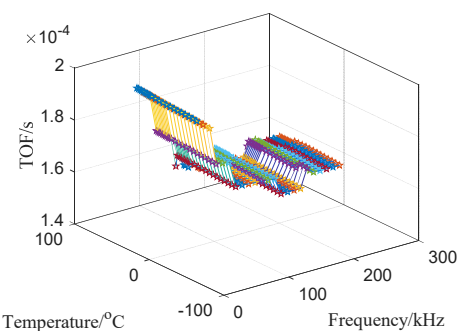

a) Scatter plot-50 to $250 \mathrm{kHz}$

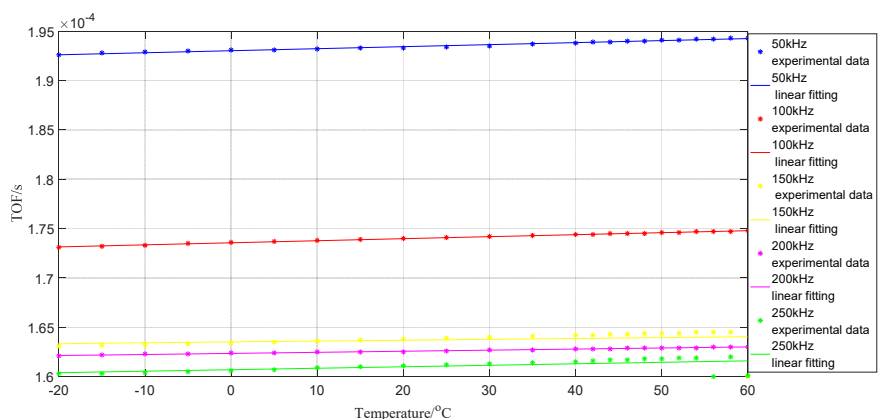

b) Plot-5 typical frequencies

Fig. 5. Influence of changing temperatures on $S_{0}$ TOF at actuator 2 to sensor 5

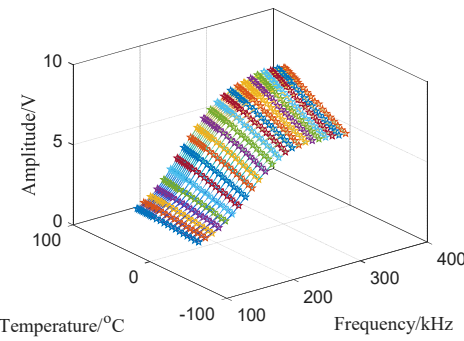

a) Scatter plot-50 to $250 \mathrm{kHz}$

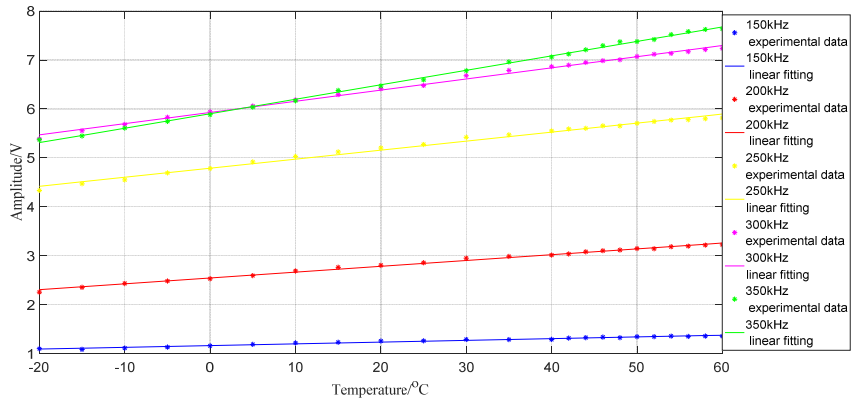

b) Plot-5 typical frequencies

Fig. 6. Influence of changing temperatures on $A_{0}$ amplitude at actuator 2 to sensor 5

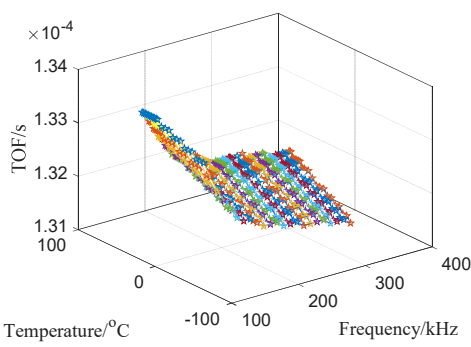

a) Scatter plot-50 to $250 \mathrm{kHz}$

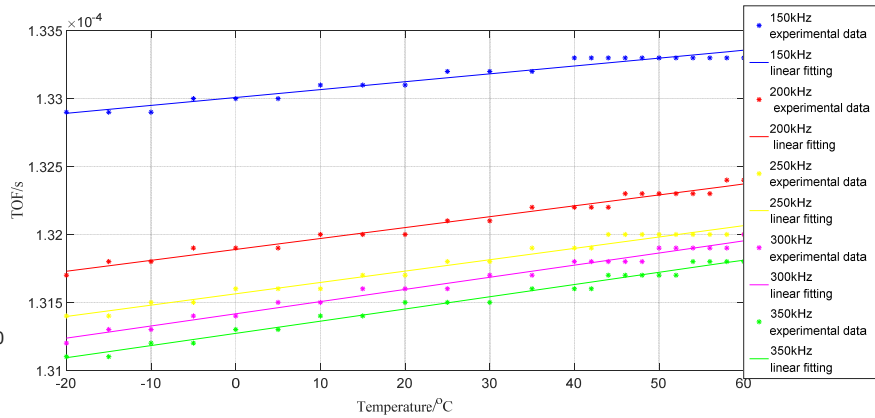

b) Plot-5 typical frequencies

Fig. 7. Influence of changing temperatures on $A_{0}$ TOF at actuator 2 to sensor 5

The results show that the amplitude and group velocity of the guided wave are approximately linearly changed with the temperatures in the range of $-20-60{ }^{\circ} \mathrm{C}$ at a certain center frequency. At the same time, according to the experimental data processing results, the amplitude of the guided wave is about $30 \%$ increases at $60{ }^{\circ} \mathrm{C}$ of a typical center frequency about $140 \mathrm{kHz}$, the linear fitting slope of $A_{0}$ mode is about $7.8 \mathrm{mV} /{ }^{\circ} \mathrm{C}$, the linear fitting slope of $S_{0}$ mode is $4.1 \mathrm{mV} /{ }^{\circ} \mathrm{C}$; while the TOF increases about $2.5 \%$ at $60{ }^{\circ} \mathrm{C}$ namely the group speed down $2.5 \%, A_{0}$ linear fitting slope is $2.25 \times 10^{-8} \mathrm{~s} /{ }^{\circ} \mathrm{C}, S_{0}$ linear fitting slope is $0.95 \times 10^{-8} \mathrm{~s} /{ }^{\circ} \mathrm{C}$.

From the above quantitative results can also be found in the other center frequencies, $A_{0}$ mode group velocity changes are larger than the $S_{0}$ mode, proved $A_{0}$ mode group velocity of temperatures changes more sensitive, while $A_{0}$ mode amplitude changes are slightly larger than the $S_{0}$ mode, proved $A_{0}$ mode amplitude of temperatures also changes more sensitive. 


\section{Conclusions}

In this paper, the effect of changing temperatures on the propagation characteristics of guided wave was studied based on the mechanism of amplitude and velocity. According to the quantitative analysis of the experimental data, the following conclusions are obtained: (1) the variation of amplitude under changing temperatures mainly depends on the properties variation of piezoelectric ceramics: amplitude of the wave propagating decreases with the increasing temperatures. The amplitude is linearly varying with the temperatures between $-20-60{ }^{\circ} \mathrm{C}$, and the amplitude changes about $30 \%$ at $60{ }^{\circ} \mathrm{C}$. (2) the change of group velocity of guided wave mainly depends on the material properties changes of the substructures: the velocity of guided waves decreases with the increasing changing. The group velocity is linearly varying with the temperatures between $-20-60{ }^{\circ} \mathrm{C}$, and the change of group velocity is about $2.5 \%$ at $60{ }^{\circ} \mathrm{C}$.

According to the above basic simple structure and changing temperatures effect, the influence law of temperatures on guided wave propagation characteristics can be further analyzed. At the same time, according to the influence mechanism of in-depth discussion under various conditions and with the complex structures in the actual engineering application should be studied.

\section{References}

[1] Dib G., Udpa L. Design and performance of optimal detectors for guided wave structural health monitoring. Structural Health Monitoring, Vol. 15, Issue 1, 2016, p. 21-37.

[2] Qiu L., Yuan S., Wang Q. Design and experiment of PZT network-based structural health monitoring scanning system. China Aviation Journal, Vol. 22, Issue 5, 2009, p. 505-512.

[3] Giurgiutiu V. Structural health monitoring. Journal of Intelligent Material Systems and Structures, Vol. 16, 2005, p. 291-305.

[4] Yuan S., Ren Y., Qiu L., Mei H. A multi-response-based wireless impact monitoring network for aircraft composite structures. IEEE Transactions on Industrial Electronics, Vol. 63, Issue 12, 206, p. 7712-7722.

[5] Qiu L., Liu B., Yuan S., Su Z. Impact imaging of aircraft composite structure based on a model-independent spatial-wavenumber filter. Ultrasonics, Vol. 64, 2016, p. 10-24.

[6] Lee H. J., Saravanos D. A. The effect of temperature dependent material nonlinearities on the response of piezoelectric composite plates. NASA/TM-97-206216, 1997.

[7] Hooker M. W. Properties of PZT-Based Piezoelectric Ceramics Between -150 and $250{ }^{\circ} \mathrm{C}$. NASA Langley Technical Report Server, 1998.

[8] Raghavan A., Cesnik C. E. Effects of elevated temperature on guided-wave structural health monitoring. Journal of Intelligent Material Systems and Structures, Vol. 19, Issue 12, 2008, p. 1383-1398.

[9] Lanza D. S. F., Salamone S. Temperature effects in ultrasonic Lamb wave structural health monitoring systems. The Journal of the Acoustical Society of America, Vol. 124, Issue 1, 2008, p. $161-174$

[10] Marzani A., Salamone S. Numerical prediction and experimental verification of temperature effect on plate waves generated and received by piezoceramic sensors. Mechanical Systems and Signal Processing, Vol. 30, 2012, p. 204-217.

[11] Roy S., Ladpli P., Lonkar K., Chang F. K. Structural damage detection using ultrasonic guided waves under varying ambient temperature and loading environments. Proceedings of the 9th international workshop on structural health monitoring, 2017.

[12] Lonkar K. Modeling of Piezo-Induced Ultrasonic Wave Propagation for Structural Health Monitoring. Ph.D. Thesis, Stanford, 2013.

[13] Dodson J. C., Inman D. J. Thermal sensitivity of Lamb waves for structural health monitoring applications. Ultrasonics, Vol. 53, Issue 3, 2013, p. 677-685. 